\title{
Hydrodynamic pressures on sloping dams during earthquakes. Part 2. Exact theory
}

\author{
By ALLEN T. CHWANG
}

Division of Engineering and Applied Science, California Institute of Technology, Pasadena

(Received 5 October 1977 and in revised form 6 December 1977)

The equations for the earthquake forces on a rigid dam with an inclined upstream face of constant slope are solved exactly by two-dimensional potential-flow theory. The distribution of the hydrodynamic pressure along the upstream face and the total horizontal, vertical and normal loads on the dam are computed from the integral solutions. The results obtained from the exact theory are compared with those derived from the momentum-balance method and there is reasonable agreement.

\section{Introduction}

An important factor in the design of dams in seismic regions is the action of hydrodynamic pressure exerted on the upstream face of the dam as a result of earthquake ground movements. In part 1 of this study (Chwang \& Housner 1978), the twodimensional problem of the added-mass effect of horizontal acceleration of a rigid dam with an inclined upstream face of constant slope was solved analytically by adopting von Kármán's (1933) momentum-balance approach. The distribution of the hydrodynamic pressure along the sloping dam was determined, and explicit analytical formulae for evaluating the total horizontal, vertical and normal loads were also presented. It was noted that the normal force coefficient remains practically constant at around $\mathbf{0 . 5}$ for all slopes.

The objective of this paper is to present an integral solution for the earthquake force on a rigid sloping dam based on the exact two-dimensional potential-flow theory. The results based on this exact theory are then compared with those derived from the momentum-balance method. The two methods are found to be in reasonable agreement, especially for the total force exerted on the face of the dam.

\section{Two-dimensional potential-flow theory}

As in part 1 of this study, the upstream face of the dam is assumed to have a constant slope $1 / \beta$ (see figure 1). If the angle between this interface and the horizontal is $\theta=\alpha \pi$, then

$$
\beta=\cot \alpha \pi \text {. }
$$

The fluid in the reservoir is bounded below by a horizontal bottom at $y=0$ and above by a free surface at $y=h$. The dam is assumed to be rigid and to undergo a constant horizontal acceleration $a_{0}$ in the $x$ direction of sufficiently short duration that the perturbation of the free surface is negligible. 

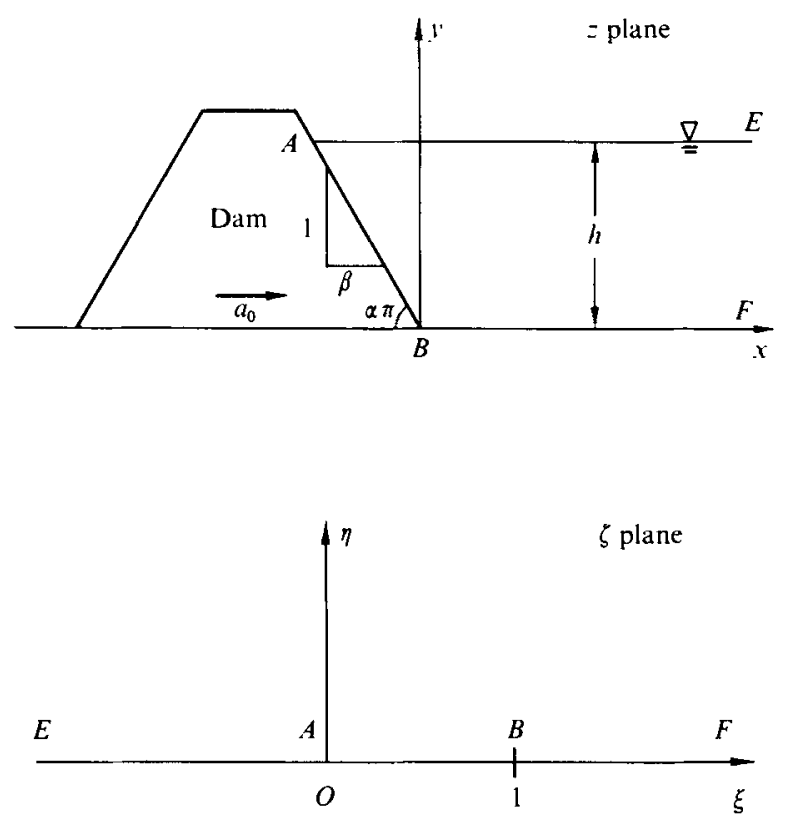

Figure 1. The reservoir occupied by the fluid in the physical $(z)$ plane is mapped conformally into the upper half of the $\zeta$ plane.

With $z=x+i y$, the conformal mapping

$$
z=\frac{h}{\pi} \int_{1}^{\zeta}\left(\frac{\zeta}{\zeta-1}\right)^{a} \frac{d \zeta}{\zeta}
$$

given by the Schwarz-Christoffel theory, transforms the upper half of the $\zeta$ plane $(\zeta=\xi+i \eta)$ into the region occupied by the fluid (see figure 1). The points $A$ and $B$ in the physical $(z)$ plane are mapped into $\zeta=0$ and +1 respectively. The points at infinity on the free surface $(E)$ and at the reservoir bottom $(F)$ are mapped into the points at infinity in the $\zeta$ plane along the negative and positive real axes respectively. The branch in the $\zeta$ plane is so chosen that the argument of the variable in brackets in $(2)$ vanishes as $\zeta$ goes to infinity.

Since the fluid in the reservoir is assumed to be incompressible and inviscid, the hydrodynamic pressure $p$ (in addition to the hydrostatic pressure) caused by the horizontal acceleration $a_{0}$ of the dam satisfies the Laplace equation

$$
\nabla^{2} p=0 .
$$

If we introduce the complex-conjugate function $q$ with respect to $p$, we can construct an analytic function

$$
f(z) \equiv p+i q
$$

which is also analytic in the transformed variable $\zeta$. On the free surface, which corresponds to the negative real axis in the $\zeta$ plane, the pressure $p$ vanishes. At the reservoir bottom, which corresponds to $\zeta>1$ on the positive real axis, we have $\partial p / \partial n=0$, which means that $q$ is a constant. Without loss of generality we may assume $q=0$ for $\zeta>1$ along the positive real axis. By contrast, on the sloping upstream face of the dam $\partial p / \partial n$ takes the constant value $-\rho a_{0} \sin \alpha \pi$ in the $z$ plane. 
Now, if $s$ represents the distance along that sloping face measured from the bottom, the Cauchy-Riemann condition $\partial p / \partial n=\partial q / \partial s$ gives $q=-\rho a_{0} s \sin \alpha \pi$ along that sloping face, which corresponds to the line segment $0<\zeta<1$ in the $\zeta$ plane. Therefore, along the real axis in the $\zeta$ plane, we have

$$
\begin{gathered}
\operatorname{Re} f(\zeta)=0 \quad(-\infty<\xi<0), \\
\operatorname{Im} f(\zeta)=\left\{\begin{array}{cc}
-\rho a_{0} s \sin \alpha \pi \quad(0<\xi<1), \\
0 \quad(1<\xi<\infty),
\end{array}\right.
\end{gathered}
$$

where the distance $s$ is given by (2) as

$$
s(\xi)=\frac{h}{\pi} \int_{\xi}^{1}\left(\frac{t}{1-t}\right)^{\alpha} \frac{d t}{t} \quad(0<\xi<1) .
$$

Equation (5) is a mixed boundary condition for $f(\zeta)$. However, if we introduce a new analytic function $g(\zeta)$ by

$$
g(\zeta)=\zeta^{-\frac{1}{2}} f(\zeta)
$$

where the positive branch is taken for the square-root function, (5) becomes an 'unmixed' boundary condition for $g(\zeta)$. Thus for real $\zeta$ we have

$$
\operatorname{Im} g(\zeta)= \begin{cases}-\rho a_{0} \zeta^{-\frac{1}{2}} s(\zeta) \sin \alpha \pi & (0<\zeta<1) \\ 0 \quad \text { (otherwise) } & \end{cases}
$$

An analytic function $g(\zeta)$ which is regular in the upper half $\zeta$ plane and vanishes at infinity can be obtained from the Poisson integral formula:

$$
g(\zeta)=\frac{1}{\pi} \int_{-\infty}^{\infty} \frac{\operatorname{Im} g(\xi) d \xi}{\xi-\zeta}
$$

Substituting (7) and (8) into (9), we have

$$
f(\zeta)=-\frac{1}{\pi} \rho a_{0} \zeta^{\frac{1}{2}} \sin \alpha \pi \int_{0}^{1} \frac{s(\xi) d \xi}{\xi^{\frac{1}{2}}(\xi-\zeta)} .
$$

The hydrodynamic pressure on the dam is the real part of $f(\zeta)$ for $0<\zeta<1$. By (6) and (10), we obtain

$$
p(\xi)=\frac{1}{\pi^{2}} \rho a_{0} h \sin \alpha \pi \mathrm{P} \int_{0}^{1}\left(\frac{t}{1-t}\right)^{\alpha} \log _{e}\left|\frac{t^{\frac{1}{2}}+\xi^{\frac{1}{2}}}{t^{\frac{1}{2}}-\xi^{\frac{1}{2}}}\right| \frac{d t}{t} \quad(0<\xi<1),
$$

where $\mathrm{P}$ denotes the Cauchy principal value. Equation (11a) can be expressed in a different form for computational purposes. On differentiating $(11 a)$ with respect to $\xi$, we have

$$
\frac{d p(\xi)}{d \xi}=\frac{\rho a_{0} h \sin \alpha \pi}{\pi^{2} \xi^{\frac{1}{2}}} \mathrm{P} \int_{0}^{1}\left(\frac{t}{1-t}\right)^{\alpha} \frac{d t}{t^{\frac{1}{2}}(t-\xi)}
$$

The integrand in (12a) has three branch points in the complex $t$ plane, namely the origin, $t=1$, and the point of infinity. By contour integration around the branch cut which connects these three branch points and along a circle of large radius, we can simplify $(12 a)$ to

$$
\frac{d p(\xi)}{d \xi}=\frac{\rho a_{0} h}{\pi^{2}}\left[\frac{1}{\xi^{\frac{1}{2}}} \int_{0}^{\infty}\left(\frac{t}{t+1}\right)^{\alpha} \frac{d t}{t^{\frac{1}{2}}(t+\xi)}-\frac{\pi \cos \alpha \pi}{\xi}\left(\frac{\xi}{1-\xi}\right)^{\alpha}\right]
$$


Integrating (12b) and making use of $(6)$ and some simple transformations, we have

$$
p(\xi)=\rho a_{0} h\left[\frac{4}{\pi^{2}} \int_{0}^{\frac{1}{2} \pi}\left(\frac{\xi}{\xi+\tan ^{2} \theta}\right)^{\alpha} \frac{\theta d \theta}{\sin \theta \cos \theta}-\cot \alpha \pi+\frac{\cos \alpha \pi}{h} s(\xi)\right] \quad(0<\xi<1) .
$$

The total normal force on the sloping dam can be found by integrating (11) in the physical ( $z$ ) plane:

$$
F_{n}=\int_{0}^{h / \sin \alpha \pi} p d s=\frac{h}{\pi} \int_{0}^{1}\left(\frac{\xi}{1-\xi}\right)^{\alpha} \frac{p(\xi)}{\xi} d \xi
$$

By means of $(11 b),(13)$ reduces to

$$
F_{n}=C_{n} \rho a_{0} h^{2}
$$

where the normal force coefficient $C_{n}$ is given by

$$
C_{n}=\frac{4}{\pi^{3}} \int_{0}^{1}\left(\frac{\xi}{1-\xi}\right)^{\alpha} \frac{d \xi}{\xi} \int_{0}^{\frac{1}{2} \pi}\left(\frac{\xi}{\xi+\tan ^{2} \theta}\right)^{\alpha} \frac{\theta d \theta}{\sin \theta \cos \theta}-\frac{1}{2} \frac{\cos \alpha \pi}{\sin ^{2} \alpha \pi} .
$$

The total horizontal and vertical forces on the upstream face of the dam follow from (14):

$$
\begin{array}{ll}
F_{x}=C_{x} \rho a_{0} h^{2}, & C_{x}=C_{n} \sin \alpha \pi, \\
F_{y}=C_{y} \rho a_{0} h^{2}, & C_{y}=C_{n} \cos \alpha \pi .
\end{array}
$$

These expressions were evaluated by means of a digital computer.

\section{Results and discussion}

The pressure coefficient $C_{p}$, defined by

$$
p=C_{p} \rho a_{0} h
$$

can be obtained along the upstream face of the dam as a function of the distance $s$ measured from the bottom of the dam by using (6) and $(11 b)$ with $\xi$ as a parameter. In particular, when $\alpha=\frac{1}{2}$, the pressure coefficient at the dam base becomes

$$
C_{p}=8 \pi^{-2} G=0.7425 \quad\left(\alpha=\frac{1}{2}, \quad \xi=1\right),
$$

where $G=0.915965 \ldots$ is Catalan's constant. This result agrees exactly with Westergaard's (1933) result for dams with a vertical upstream face. The detailed pressure distribution along the sloping dam computed from (6) and $(11 b)$ is shown in figure 2, in which $C_{p}$ is plotted $v s$. the vertical distance $y / h$ for several inclination angles $\theta(\alpha \pi)$ between $15^{\circ}$ and $90^{\circ}$. The corresponding results derived in part 1 by the momentum method are shown as dashed lines in figure 2 for comparison. We can see from figure 2 that, for any fixed height $y / h$, both the pressure based on the momentum method and the pressure based on the exact theory decrease as the inclination angle $\theta$ decreases. However, for fixed values of $\theta$, the momentum method indicates that the maximum pressure occurs at the base of the dam, whereas the exact theory gives the maximum pressure at some distance above the dam base except when $\theta=90^{\circ}$, when the pressure reaches its maximum value at the base of the dam in both the exact and the approximate theory. In general, the agreement between the momentum method and the exact theory is fairly good, and it becomes even better when we consider the total horizontal, vertical and normal forces. The horizontal, vertical and normal force 


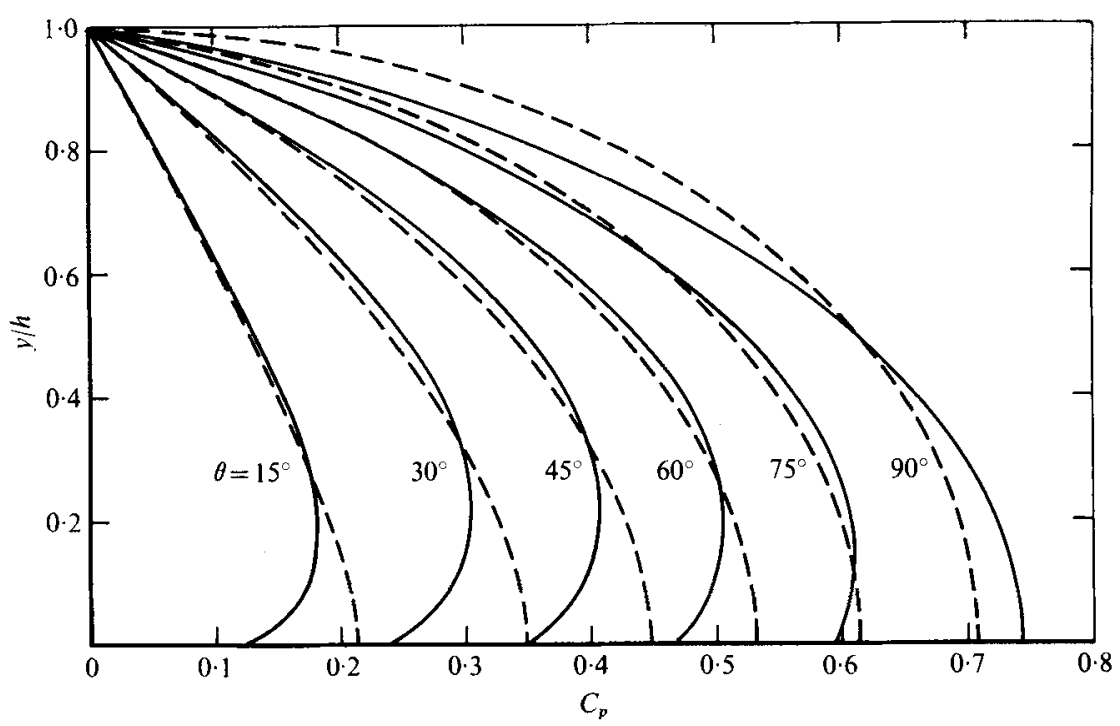

Figure 2. The pressure distributions on the upstream face of a dam determined from the exact theory (solid curves) and from the momentum method (dashed curves).

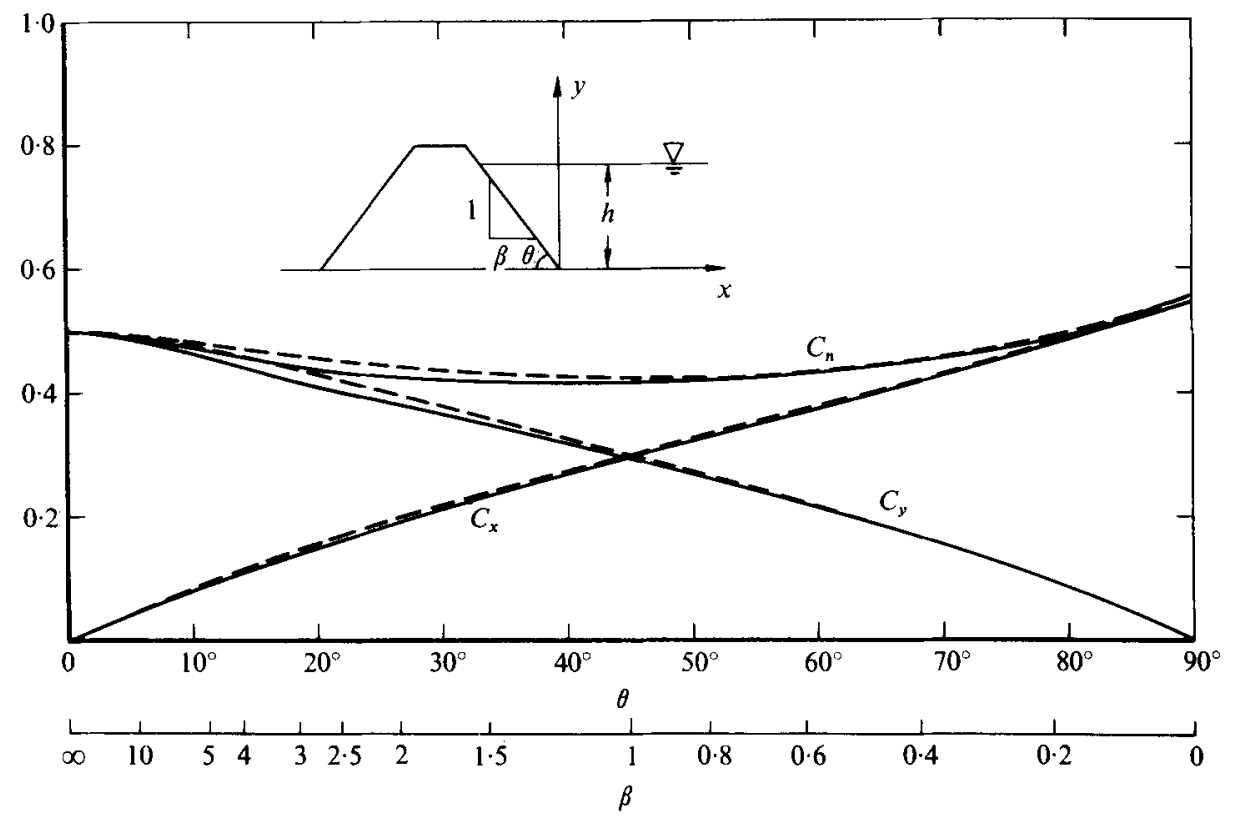

Figure 3. The horizontal, vertical and normal force coefficients determined from the exact theory (solid curves) and from the momentum method (dashed curves) $v s$. the inclination angle $\theta$ or the reciprocal of the slope $\beta$.

coefficients $C_{x}, C_{y}$ and $C_{n}$, given by (15), (16) and (14b) respectively, are plotted in figure 3 as solid lines $v s$. the angle $\theta$ or the reciprocal of the slope $\beta$. The corresponding results from the momentum method are also plotted in figure 3 , as dashed lines. When the upstream face of the dam becomes vertical, the exact theory gives

$$
C_{x}=C_{n}=0.543, \quad C_{\nu}=0 \quad\left(\theta=90^{\circ}\right),
$$


which is the same as Westergaard's (1933) result, while the momentum method gives

$$
C_{x}=C_{n}=0.555, \quad C_{y}=0 \quad\left(\theta=90^{\circ}\right)
$$

which is the same as von Kármán's (1933) result. The difference between the values given by the exact theory and by the momentum method is less than $3 \%$. For arbitrary values of $\theta$ between 0 and $90^{\circ}$ the maximum difference between the force coefficients given by the exact theory and the momentum method is about $4.5 \%$, the momentum method being on the high side. From figure 3 we may also note that the approximate rule

$$
C_{n} \doteqdot 0 \cdot 5 \quad\left(0 \leqslant \theta \leqslant 90^{\circ}\right)
$$

given in part 1 is still valid. This approximation may be very useful to dam engineers for making quick estimates for preliminary designs.

The author is indebted to Professor Sir James Lighthill, Professor Theodore Y. Wu and Professor George W. Housner for valuable suggestions and comments. This work was sponsored by the National Science Foundation, under Grant PFR 77-16085.

\section{REFERENCES}

Chwang, A. T. \& Housner, G. W. 1978 Hydrodynamic pressures on sloping dams during earthquakes. Part 1. Momentum method. J. Fluid Mech. 87, 335-341.

KÁRmán, T. voN 1933 Discussion of water pressures on dams during earthquakes. Trans. A.S.C.E. 98, 434-436.

WeStergaARD, H. M. 1933 Water pressures on dams during earthquakes. Trans. A.S.C.E. 98, 418-433. 